\title{
Cáncer colorrectal hereditario: presentación sincrónica de cáncer colorrectal y colangiocarcinoma en un paciente con poliposis adenomatosa familiar
}

\author{
Hereditary colorectal cancer: synchronous presentation of colorectal cancer \\ and cholangiocarcinoma in a patient with familial adenomatous polyposis
}

Alberto Félix Chapa-Lobo, ${ }^{*}$ Luis Enrique Salgado-Cruz, ${ }^{*}$ Alan Alejandro Garza-Cantú, ${ }^{*}$ Mauricio David Castaño-Eguía, Fernando Rodríguez-Sotelo, ${ }^{*}$ Roy Ismael Villafranca-Andino*

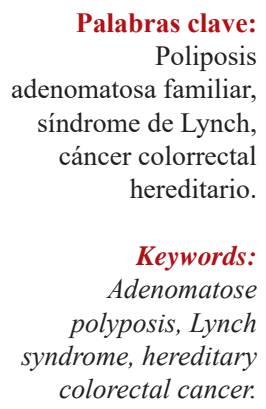

* Departamento de Cirugía General, Christus Muguerza Hospital Alta Especialidad. Monterrey, Nuevo León. Aceptado: 20/08/2019

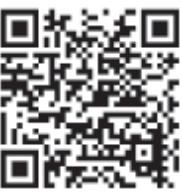

\section{RESUMEN}

Los síndromes de poliposis gastrointestinales se caracterizan por la presencia de múltiples pólipos en el tubo digestivo que afectan preferentemente el colon y recto. Representan alrededor de 5\% de los tipos de cáncer colorrectal, siendo los más comunes la poliposis adenomatosa familiar y el cáncer de colon no polipósico hereditario (síndrome de Lynch). Son un grupo de enfermedades de escasa incidencia con características muy variadas que precisan una correcta individualización para su tratamiento más adecuado. Dentro de los síndromes genéticos hay presentaciones con variaciones moleculares típicas que encasillan a los grupos más frecuentes; sin embargo, existe la posibilidad de que dichos síndromes muestren similitudes genéticas cuya predominancia determine la evolución y presentación de la enfermedad.

\section{ABSTRACT}

The gastrointestinal polyposis syndromes are characterized by the presence of multiple polyps in the digestive tract, affecting the colon and rectum in the most cases. Recognized family syndromes represent around 5\% of colorectal cancers, the most common being familial adenomatose polyposis (FAP) and hereditary nonpolyposis colon cancer (Lynch syndrome). They are a group of diseases of low incidence with very varied characteristics, which require a correct individualization for their most appropriate treatment. Within the genetic syndromes, there are presentations with typical molecular variations that typecast the most frequent groups, however there is the possibility that these syndromes present genetic similarities whose predominance will determine the evolution and presentation of the disease.

\section{INTRODUCCIÓN}

$\mathrm{A}^{\mathrm{n}}$ nivel mundial, el cáncer colorrectal (CCR) representa la tercera neoplasia maligna más común y la cuarta causa principal de mortalidad relacionada con el cáncer y cuyos síndromes familiares reconocidos corresponden a alrededor de 5\% de los casos. ${ }^{1,2}$ Existen dos amplias clases de cáncer colorrectal hereditario, según la ubicación predominante del cáncer: distal y proximal. La poliposis adenomatosa familiar (PAF) y la mayoría de los casos esporádicos pueden considerarse un paradigma para la clase distal, mientras que el cáncer colorrectal no polipósico hereditario (síndrome de Lynch) representa más claramente la clase proximal. 3,4

La PAF es un síndrome autosómico dominante causado por una mutación germinal del gen de la poliposis adenomatosa coli. ${ }^{5}$ Afecta a 1:10,000 personas y representa aproximadamente $1 \%$ del cáncer colorrectal. Su característica principal es la aparición de más de 100

Citar como: Chapa-Lobo AF, Salgado-Cruz LE, Garza-Cantú AA, Castaño-Eguía MD, Rodríguez-Sotelo F, Villafranca-Andino RI. Cáncer colorrectal hereditario: presentación sincrónica de cáncer colorrectal y colangiocarcinoma en un paciente con poliposis adenomatosa familiar. Cir Gen. 2020; 42(1): 50-56. doi: 10.35366/92712 
pólipos adenomatosos colorrectales, pudiendo llegar a ser miles, iniciando en edad joven, con riesgo de cáncer cercano a 100\% si no recibe tratamiento oportuno. La mayoría de los casos comienzan como pólipos colónicos adenomatosos benignos. $2,6,7$

La complejidad de las posibles presentaciones clínicas de estos síndromes incluye, además de los trastornos colorrectales severos o atenuados, diversas manifestaciones extracolónicas como poliposis gástrica y duodenal y tumores desmoides, que pueden requerir tratamiento endoscópico o quirúrgico adicional que complica el proceso terapéutico e impone una vigilancia continua incluso cuando la enfermedad colorrectal es erradicada. ${ }^{8}$

\section{PRESENTACIÓN DE CASO}

Se trata de un hombre de 56 años de edad, bajo tratamiento médico por hipertensión arterial de larga evolución y cardiopatía isquémica tratada con colocación de stent coronario hace dos años, antecedente heredofamiliar de cáncer de colon en dos familiares de primer grado menores de 50 años. Acude a valoración externa por historia de tres meses de evolución con sangrado de tubo digestivo bajo (STDB) acompañado de síndrome anémico, se realiza colonoscopia observando poliposis múltiple (más de 200 pólipos), con lesiones de aspecto neoplásico en colon sigmoides y colon transverso (Figura 1). No se encontraron lesiones cutáneas u óseas anormales ni tampoco se identificó la presencia de hipertrofia del pigmento del epitelio retiniano.

Se complementó su estudio con endoscopia de tubo digestivo alto observando superficie micronodular difusa en porción alta del cuerpo del estómago, sin pólipos duodenales (Figura 2), y tomografía computarizada toracoabdominal con contraste iv, que reveló tumoración de colon transverso con infiltración de grasa pericolónica, la cual condiciona obstrucción parcial, así como la presencia de adenopatías paraaórticas (Figura 3). En los estudios de laboratorios se observó una hemoglobina: $9.0 \mathrm{~g} /$ dl, proteínas totales: $5.8 \mathrm{~g} / \mathrm{dl}$, albumina $3.0 \mathrm{~g} /$ dl y antígeno carcinoembrionario: $131 \mathrm{ng} / \mathrm{ml}$.

Durante el segundo día de internamiento, el STDB continúa con disminución de la hemoglobina a $7.0 \mathrm{~g} / \mathrm{dl}$ y agregándose cuadro de oclusión intestinal, por lo que se decide programar para cirugía.

Se realiza colectomía total con ileostomía terminal por laparoscopia de cuatro puertos manoasistida, encontrando múltiples implantes peritoneales y hepáticos, de los cuales se tomaron biopsias. En el postoperatorio, el paciente desarrolla íleo adinámico que se resuelve hasta transcurridos 18 días con nutrición parenteral, posteriormente toleró la vía oral, con ileostomía funcional, previa colocación de catéter Port-aCath a su egreso.

El departamento de patología reportó más de 200 pólipos adenomatosos tubulares con displasia de bajo grado, así como la presencia de adenocarcinoma tipo intestinal con
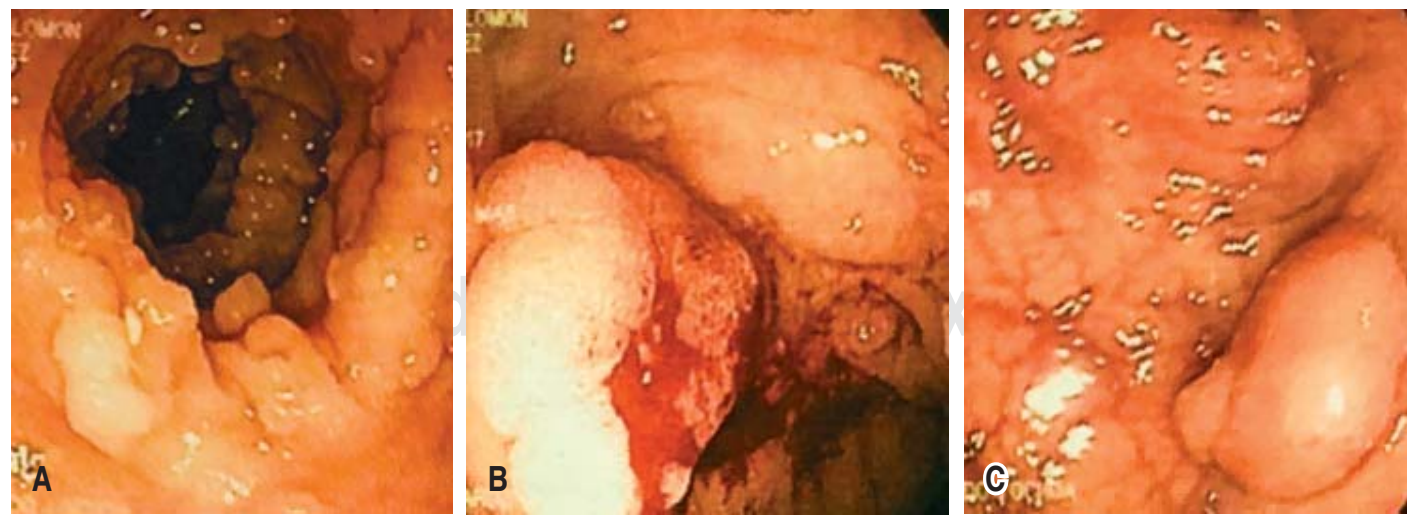

Figura 1: Colonoscopia donde se observa poliposis múltiple (A), con lesiones de aspecto neoplásico en colon transverso (B) y colon sigmoides $(\boldsymbol{C})$. 


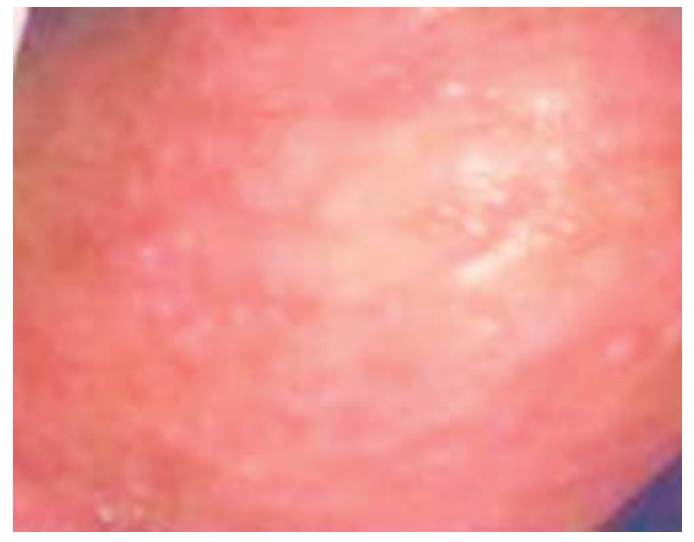

Figura 2: Endoscopia: superficie micronodular difusa en porción alta del cuerpo del estómago, sin pólipos duodenales.

componente mucinoso en colon transverso y sigmoides. La biopsia hepática reveló colangiocarcinoma (CCA) tipo I. Se realizó panel de inmunohistoquímica por inestabilidad microsatelital: MLHI, MSH2, MSH6 y PMS2 con expresión nuclear conservada, y pérdida de la expresión nuclear del gen APC, prueba molecular de identificación de mutación del gen KRAS negativa, con CK7 (+), CK9 (+) y CK20 (-) en biopsia hepática, sugiriendo PAF asociada con colangiocarcinoma primario (Figura 4).

El paciente fue readmitido para recibir adyuvancia con quimioterapia a base de FOLFOX + bevacizumab en seis ciclos, con buena tolerancia, actualmente asintomático.

\section{DISCUSIÓN}

La mayoría de los CCR se desarrollan a partir de lesiones preneoplásicas benignas: pólipos adenomatosos o adenomas. Vogelstein propuso un modelo multistep de carcinogénesis para el desarrollo del CCR que describe la progresión de un adenoma benigno a un carcinoma maligno a través de una serie de etapas histológicas bien definidas, lo que se conoce como el modelo de secuencia adenoma-carcinoma, de acuerdo con esto se entiende que la etiología del CCR es multifactorial y es probable que implique las acciones de genes en múltiples niveles, entre los que han sido implicados p53, APC, factor de crecimiento transformante (TGF)- $\beta$, SMAD,
MLH1, MSH2, MSH6, PMS2, AXIN, STK11, PTEN, DCC y KRAS. ${ }^{9}$

En 1991 se descubrió el gen responsable de la PAF, llamado Adenomatous Polyposis Coli o gen APC. ${ }^{10}$ Se han descubierto más de 300 diferentes mutaciones en dicho gen que pueden causar este tipo de poliposis (Anexo 1), se demostró que el riesgo de desarrollar manifestaciones específicas de PAF, así como la gravedad de la enfermedad en el intestino grueso están relacionados con el tipo de mutación genética. Las más graves se observan con mayor frecuencia en las mutaciones entre los codones 1250 y 1464. Esto implica un comienzo temprano con síntomas (dolor abdominal, diarrea, sangre en las heces) incluso antes de los 20 años de edad, un alto número de pólipos en colon y recto y una progresión
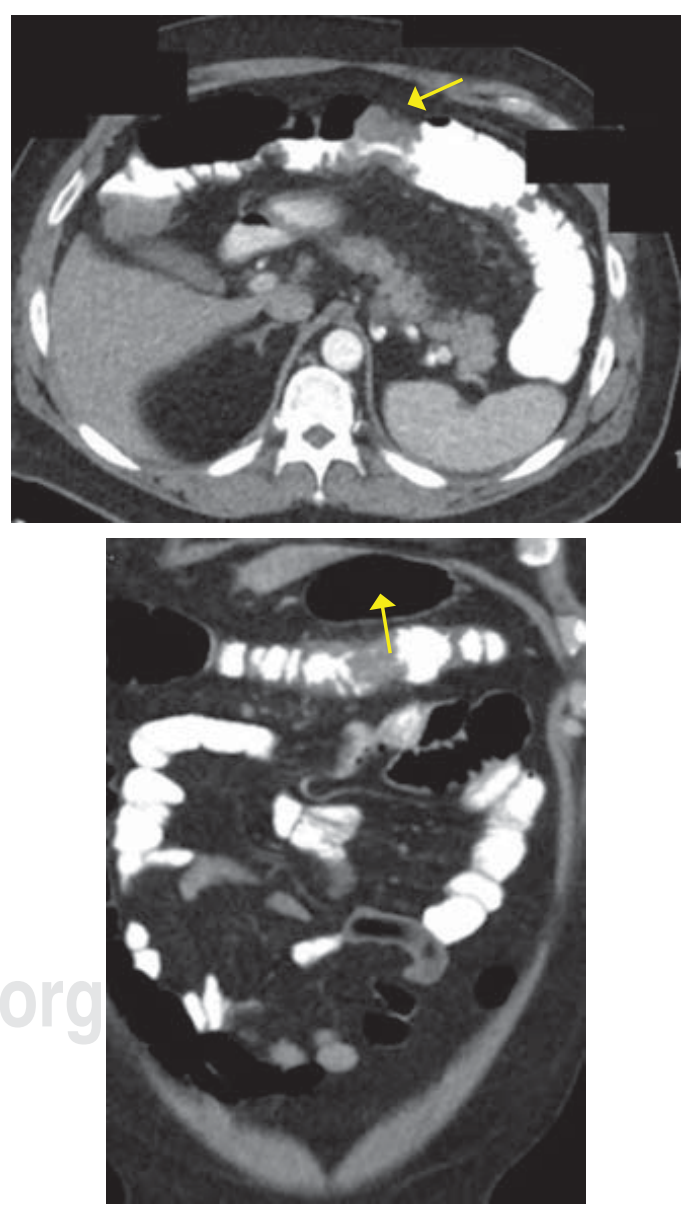

Figura 3: Tumoración en colon transverso parcialmente oclusiva (flecha amarilla). 

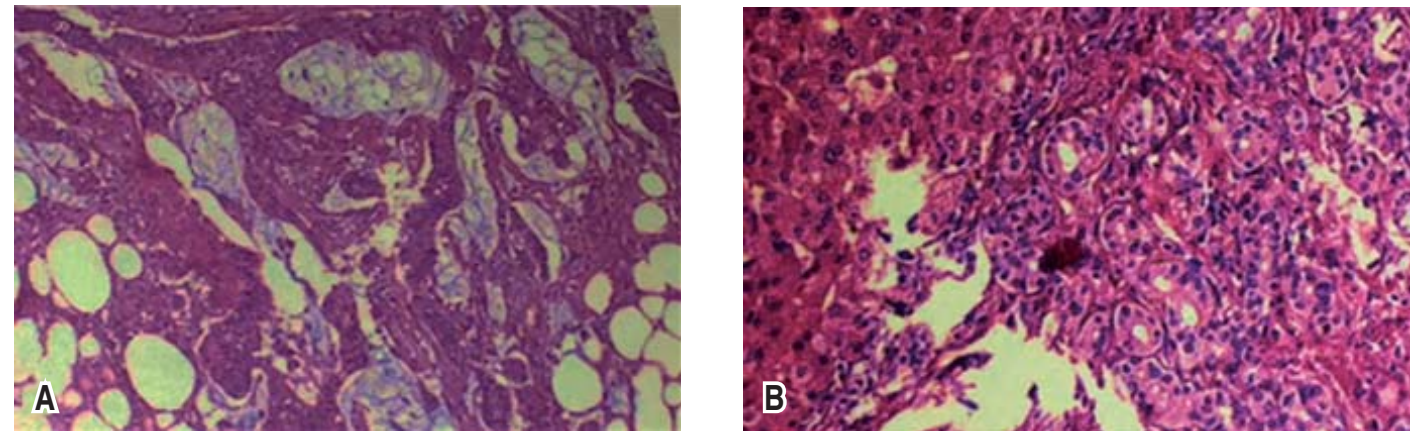

Figura 4: (A) Adenocarcinoma colónico mucosecretor con células en anillo de sello, (B) Adenocarcinoma en hígado bien diferenciado.

temprana a malignidad. Aunque clínicamente comprobado, la PAF no tiene una mutación genética reconocible, existe una gran cantidad de manifestaciones extracolónicas de la PAF, entre las que destacan osteomas, quistes epidermoides, tumores desmoides, afección gastroduodenal, hipertrofia congénita del epitelio pigmentado de la retina, entre otros. ${ }^{11}$

El manejo de este tipo de enfermedades o síndromes hereditarios debe iniciarse con un consejo genético para informar del tipo de patología, de la mejor terapéutica y del seguimiento necesario, disminuyendo así la morbimortalidad atribuible a estos síndromes. La prevención se verá favorecida por la identificación específica de la mutación causal de la línea germinal en la familia del paciente, lo que clasifica el riesgo y marca la pauta para el plan terapéutico y de vigilancia. Actualmente, la cirugía profiláctica es obligatoria; sin embargo, el tipo de técnica quirúrgica dependerá de la gravedad de las manifestaciones y del genotipo presentado. $^{12,13}$

El CCA es un tumor maligno que surge del epitelio biliar en cualquier parte del sistema del conducto biliar, desde los conductos biliares hasta la ampolla de Vater. Se asocia a menudo con la inactivación de genes supresores de tumores, por ejemplo, p53, SMAD4, Bcl-2 y p16. También se han descrito mutaciones en oncogenes, incluyendo KRAS, p53, c-ErbB-2 y c-Neu. Aunque las mutaciones pueden conducir a cambios fenotípicos detectables, los perfiles moleculares en la citología biliar no tienen en la actualidad un papel establecido de diagnóstico o pronóstico. ${ }^{14}$
Distinguir el CCA intrahepático del adenocarcinoma metastásico y otros tumores hepáticos primarios puede ser difícil. La diferenciación requiere en particular de las metástasis del tubo digestivo que a menudo no se pueden realizar por histología. Otras modalidades especialmente de imagen son esenciales. Pueden ser útiles los paneles de inmunohistoquímica que incluyen CK7, CK19, CK20, CDX-2, TTF-1, receptores de estrógeno/progesterona y PSA, dependiendo del contexto clínico. Los CCA suelen ser CK7 positivos y CK20 negativos, ${ }^{15,16}$ como los que presentó nuestro paciente.

En la actualidad no existen marcadores séricos específicos para neoplasias de la vía biliar en pacientes sanos o portadores de polimorfismos genéticos. Existen estudios prometedores de marcadores como diferentes mucinas, interleucina-6, ácido siálico y metaloproteinasas de matriz utilizados en combinación, pero se necesitan más investigaciones. ${ }^{17}$

El estándar de oro ideal para la prevención de complicaciones o retrasar el desarrollo de cáncer colorrectal sería una técnica genética que permitiera la eliminación del gen enfermo y la implantación de un gen libre de enfermedad. En este momento, esto sigue siendo pura especulación. La mayor parte de la investigación trata sobre los sistemas para el cribado de las mutaciones de la línea germinal en el gen de la poliposis adenomatosa coli que predispone a la susceptibilidad y enfermedad en la poliposis adenomatosa familiar. Hoy en día existen sistemas técnicos que detectan las mutaciones en el gen APC, las cuales podrían ser útiles en el diagnóstico molecular de casos 
presintomáticos en familias con $\mathrm{PAF} ;{ }^{18}$ sin embargo, hay otras formas interesantes de abordar el problema de una evaluación de riesgo de portador presintomática en la poliposis adenomatosa familiar como el uso combinado de marcadores moleculares y biomarcadores, siendo necesaria la comprensión detallada del proceso de carcinogénesis. ${ }^{19}$

\section{CONCLUSIÓN}

La poliposis adenomatosa familiar puede no ser considerada una sola entidad de enfermedad con guías estándar para el tratamiento quirúrgico. Sin embargo, la colectomía profiláctica después de la manifestación de pólipos, pero antes del desarrollo del cáncer colorrectal, sigue siendo la prevención más efectiva. En la actualidad, las técnicas de análisis genético de refinación y las nuevas terapias dirigidas con la posibilidad de identificar la mutación que lleva el paciente (ya sea que esté relacionada con un fenotipo grave o no) han asumido un papel fundamental en la indicación del tipo de tratamiento quirúrgico en términos de radicalidad. Algunas herramientas bioinformáticas pretenden predecir la sensibilidad del tumor a los fármacos en función de su característica molecular, así como en el seguimiento a corto y largo plazo tanto del paciente como de sus familiares de primer grado. ${ }^{20,21}$

Las opciones quirúrgicas son la proctocolectomía con ileostomía terminal, la colectomía subtotal con anastomosis ileorrectal y la proctocolectomía restauradora con reservorio ileoanal. La decisión debe basarse en la estimación del riesgo de padecer cáncer colorrectal, teniendo así que los pacientes con alto riesgo como aquéllos con más de 20 adenomas en el recto, más de 1,000 adenomas en colon, adenomas rectales mayores de $3 \mathrm{~cm}$ de diámetro o con displasia severa o en pacientes con diagnóstico confirmado de cáncer de colon o recto, la proctocolectomía restauradora con bolsa ileoanal podría ser el procedimiento de elección. ${ }^{22}$

La aplicación de diversos biomarcadores intermedios a los estudios de quimioprevención aumenta la capacidad de los investigadores para analizar los efectos de nuevos agentes quimiopreventivos en el colon y en otros órganos.

\section{REFERENCIAS}

1. Hnatyszyn A, Hryhorowicz S, Kaczmarek-Ryś M, Li E, Słomski R, Scott RJ, et al. Colorectal carcinoma in the course of inflammatory bowel diseases. Hered Cancer Clin Pract. 2019; 17: 18.

2. Byrne RM, Tsikitis VL. Colorectal polyposis and inherited colorectal cancer syndromes. Ann Gastroenterol. 2018; 31: 24-34.

3. Leoz ML, Carballal S, Moreira L, Ocaña T, Balaguer F. The genetic basis of familial adenomatous polyposis and its implications for clinical practice and risk management. Appl Clin Genet. 2015; 8: 95-107.

4. Argillander TE, Koornstra JJ, van Kouwen M, Langers AM, Nagengast FM, Vecht J, et al. Features of incident colorectal cancer in Lynch syndrome. United European Gastroenterol J. 2018; 6: 1215-1222.

5. Talseth-Palmer BA. The genetic basis of colonic adenomatous polyposis syndromes. Hered Cancer Clin Pract. 2017; 15: 5.

6. Hyer W, Cohen S, Attard T, Vila-Miravet V, Pienar C, Auth $M$, et al. Management of familial adenomatous polyposis in children and adolescents: position paper from the ESPGHAN polyposis working group. JPGN. 2019; 68: 428-441.

7. Oncolink.org [Internet] Philadelphia: OncoLink Team. [actualizado 13 jun 2017; citado 16 abr 2018]. Disponible en: https://www.oncolink.org/ cancers/gastrointestinal/colon-cancer/risk-preventionand-screening/familial-colorectal-cancers-familialadenomatous-polyposis-fap.

8. Ricker $\mathrm{CH}$. De las familias con síndromes a los genes. Las primeras caracterizaciones clínicas y genéticas de dos síndromes hereditarios con predisposición a cáncer: ¿Cuál fue el comienzo? Rev Med Condes. 2017; 28: 491-499.

9. Stoffel EM, Koeppe E, Everett J, Ulintz P, Kiel M, Osborne J, et al. Germline genetic features of young individuals with colorectal cancer. Gastroenterology. 2018; 154: 897-905.e1.

10. Russo A, Catania VE, Cavallaro A, Ficili B, Lanteri E, Tralongo $\mathrm{P}$, et al. Molecular analysis of the APC gene in sicilinan patients with familial adenomatous polyposis (F.A.P.). Int J Surg. 2014; 12: 125-129.

11. Mishra N, Hall J. Identification of patients at risk for hereditary colorectal cancer. Clin Colon Rectal Surg. 2012; 25: 67-82.

12. Mork ME, You YN, Ying J, Bannon SA, Lynch PM, Rodriguez MA, et al. High prevalence of hereditary cancer syndromes in adolescents and young adults with colorectal cancer. J Clin Oncol. 2015; 33: 3544-3549.

13. Stoffel EM, Mangu PB, Gruber SB, Hamilton SR, Kalady MF, Lau MW, et al. Hereditary colorectal cancer syndromes: American Society of Clinical Oncology clinical practice guideline endorsement of the familial risk-colorectal cancer: European Society for Medical Oncology Clinical. Practice Guidelines. J Clin Oncol. 2015; 33: 209-217.

14. Khan SA, Davidson BR, Goldin RD, Heaton N, Karani J. Guidelines for the diagnosis and treatment of cholangiocarcinoma: an update. Gut. 2012; 61: 1657-1669.

15. Songserm N, Promthet S, Pientong C, Ekalaksananan T, Chopjitt P, Wiangnon S. Gene-environment interaction involved in cholangiocarcinoma in the 
Thai population: polymorphisms of DNA repair genes, smoking and use of alcohol. BMJ Open. 2014; 4: $1-8$.

16. Cardinale V, Semeraro R, Torrice A, Gatto M, Napoli C, Bragazzi MC, et al. Intra-hepatic and extra-hepatic cholangiocarcinoma: New insight into epidemiology and risk factors. World J Gastrointest Oncol. 2010; 2: 407-416.

17. Grunnet M, Mau M. Serum tumor markers in bile duct cancer - a review. Biomarkers. 2014; 19: 437 443.

18. Sameer AS. Colorectal cancer: molecular mutations and polymorphisms. Front Oncol. 2013; 3: 114.

19. Aghagolzadeh P, Radpour R. New trends in molecular and cellular biomarker discovery for colorectal cancer. World J Gastroenterol. 2016; 22: 5678-5693.

20. Iyevleva AG, Imyanitov EN. Cytotoxic and targeted therapy for hereditary cancers. Hered Cancer Clin Pract. 2016; 14: 1-13.

21. Liu X, Newton RC, Scherle PA. Developing c-MET pathway inhibitors for cancer therapy: progress and challenges. Trends Mol Med. 2010; 16: 37-45.

22. Parrilla P, Landa JI. Cirugía AEC: manual de la Asociación Española de Cirujanos. $3^{\text {a }}$ edición. Madrid: Editorial Médica Panamericana; 2010, 47: 509-510.
Consideraciones y responsabilidad ética: Privacidad de los datos. De acuerdo a los protocolos establecidos en el centro de trabajo de los autores, éstos declaran que han seguido los protocolos sobre la privacidad de datos de pacientes preservando su anonimato. El consentimiento informado del paciente referido en el artículo se encuentra en poder del autor.

Financiamiento: No se recibió apoyo financiero para la realización de este trabajo.

Conflicto de intereses: Los autores declaran que no existe ningún conflicto de intereses en la realización del trabajo.

Correspondencia:

Alan Alejandro Garza-Cantú

Departamento de Cirugía

del Hospital Christus Muguerza.

Av. Hidalgo Núm. 2525,

Col. Obispado, 64060,

Monterrey, Nuevo León, México.

E-mail: alangarzacantu@hotmail.com 


\section{Anexo 1: Características principales de la heterogeneidad alélica de mutaciones en Adenomatous Polyposis Coli, Síndrome de Lynch y Neoplasia asociada a colitis ulcerativa crónica inespecífica (CUCI).}

\begin{tabular}{|c|c|c|c|c|}
\hline & FAP & AFAP/HFAS & HNPCC/Luynch & UCAN \\
\hline $\begin{array}{l}\text { Mean age at diagnosis of colo- } \\
\text { rectal cancer }\end{array}$ & $32-39$ & $45-55$ & $42-49$ & $40-70$ \\
\hline Distribution of cancer & Random & Mainly right colon & Mainly right colon & Mainy left colon \\
\hline No of polyps & $>100$ & $1-100$ & 1 (i.e. tumour) & \\
\hline Sex ratio (male:female) & $1: 1$ & $1: 1$ & $1.5: 1$ & $1: 1$ \\
\hline Endoscopic view of polyp & Pedunculated & Mainly flat & $\begin{array}{c}\text { Pedunculated }(45 \%) \\
\text { flat }(55 \%)\end{array}$ & None \\
\hline $\begin{array}{l}\text { Lag time (years) from early } \\
\text { adenoma to occurrence of } \\
\text { cancer }\end{array}$ & $10-20$ & 10 & 5 & $?<8$ \\
\hline $\begin{array}{l}\text { Proportion }(\%) \text { of colonic } \\
\text { cancer }\end{array}$ & 1 & 0.5 & $1-5$ & $<0.5$ \\
\hline Superficial physical stigmata & $\begin{array}{l}80 \% \text { have retinal } \\
\text { pigmentation None }\end{array}$ & None & $\begin{array}{l}\text { Only in Muir-Torre } \\
\text { syndrome }\end{array}$ & None \\
\hline Distribution of polyps & $\begin{array}{l}\text { Distal colon or } \\
\text { universal }\end{array}$ & $\begin{array}{l}\text { Main proximal to } \\
\text { splenic with rectal } \\
\text { sparing }\end{array}$ & $\begin{array}{l}\text { Mainly proxicmal to } \\
\text { splenic flexure }\end{array}$ & None \\
\hline Carcinoma histology & $\begin{array}{l}\text { More exophytic } \\
\text { growth }\end{array}$ & $\begin{array}{l}\text { Non-exophytic but } \\
\text { very variable }\end{array}$ & $\begin{array}{l}\text { Inflamation increased } \\
\text { mucin }\end{array}$ & $\begin{array}{l}\text { Mucosal ulceration } \\
\text { and inflamation }\end{array}$ \\
\hline Other associated tumours & $\begin{array}{l}\text { Duodenal adeno- } \\
\text { ma cerebral and } \\
\text { thyroid tumours, } \\
\text { medulloblastoma and } \\
\text { desmoids }\end{array}$ & Duodenal adenoma & $\begin{array}{l}\text { Endometrial ovarian, } \\
\text { gastric cancer, glio- } \\
\text { blastoma, many other } \\
\text { cancers }\end{array}$ & \\
\hline Gene (chromosome) mutation & $\begin{array}{l}\text { APC }(5 q 21) \text { distal } \\
\text { to } 5\end{array}$ & $\begin{array}{l}\text { APC }(5 q 21) \text { proxi- } \\
\text { mal to } 5\end{array}$ & $\begin{array}{l}\text { MHS2 (2p), MLH1 } \\
(3 \mathrm{p} 21), \text { PMS1 } \\
(2 \mathrm{q} 31), \text { PMS2 (7p22) }\end{array}$ & $\begin{array}{l}\text { Multiple mutations, } \\
17 p(p 53), 5 q(A P C), \\
9 p(p 16)\end{array}$ \\
\hline
\end{tabular}

$\mathrm{FAP}=$ familial adematosis polyposis coli; $\mathrm{AFAP}=$ attenuated familial adenomatous polyposis coli; HFAS = hereditary flat adenoma syndrome; HNPPC $=$ hereditary non-polyposis colon cancer; UCAN = ulcerative colitis associated neoplasia.

Tomado de: Al-Sukhni W, Aronson M, Gallinger S. Hereditary colorectal cancer syndromes: familial adenomatous polyposis and lynch syndrome. Surg Clin North Am. 2008; 88: 819-844, vii. doi: 10.1016/j.suc.2008.04.012. 